\title{
Rendezvous Simulation for On-Orbit Servicing Missions Using Advanced Robotic Technology
}

\author{
T. Boge*, H. Benninghoff* \\ * DLR e.V., Wessling, 82234, Germany \\ (Tel: ++49 8153 282485; e-mail: toralf.boge@dlr.de; heike.benninghoff@dlr.de)
}

\begin{abstract}
Increasing complexity and costs of satellite missions promote the idea of extending the operational lifetime or improving functionalities/performance of a satellite in orbit instead of simply replacing it by a new one. Further, satellites in orbit can severely be affected by aging or degradation of their components and systems as well as by consumption of available resources. These problems may be solved by satellite on-orbit servicing (OOS) missions. One of the critical issues of such a mission is to ensure a safe and reliable Rendezvous and Docking (RvD) operation performed autonomously in space. Due to the high risk associated with an RvD operation, it must be carefully analyzed, simulated and verified in detail before the real space mission can be launched. This paper describes a ground-based hardware-in-the-loop RvD simulation facility. Designed and built on 2-decade experience of RvD experiment and testing, this unique, high-fidelity simulation facility is capable of physically simulating the final approach within 25-meter range and the docking/capture process of an on-orbital servicing mission. Additionally this paper presents first results of hardware in the loop simulations for a rendezvous process to a non-cooperative target.
\end{abstract}

Keywords: Hardware in the Loop Simulation, Guidance, Navigation and Control, Vision based Navigation, On-Orbit Servicing, Rendezvous

\section{INTRODUCTION}

Meanwhile, OOS has become part of the space programs of the US, Japan, Canada and Germany. A milestone was set with the successful completion of DARPA's Orbital Express (OE) (Mulder, 2008) mission in 2007. The goal of OE was to demonstrate the ability to autonomously perform Rendezvous \& Docking (RvD) operations including maintenance activities like refuelling. In contrast to the goals of $\mathrm{OE}$, the focus of DLR is to capture non-cooperative and/or not specially prepared client spacecraft. "Non-cooperative" is understood as there is no cooperation with respect to attitude and orbit control of the client, e.g. when the client is out of operation. "Not specially prepared" means that the client satellite does not have a special docking port or retro reflectors used for vision based navigation.

\subsection{OOS missions}

Recently, several satellite projects focuses on providing onorbit servicing (OOS) capabilities in the near future. The scenarios involve a service spacecraft approaching and docking to a client satellite. The paper is based on research work performed by DLR which can be used for the following two mission scenarios.

\section{OLEV}

OLEV is a purely commercial project managed by a European consortium including a strong DLR participation.
The business case of OLEV is to build an orbital servicer which is able to dock on high value, geostationary communication satellites and to take over attitude and orbit control in order to extend the clients' lifetime after their fuel has been depleted. Beside life extension OLEV can be used for fleet management purposes like relocation to other GEO positions or disposal to graveyard orbit.

\section{DEOS}

DEOS is a robotic technology demonstration mission (Rupp, 2009). Its primary goals are to capture a tumbling noncooperative client satellite with a service spacecraft and to de-orbit the coupled configuration within a pre-defined orbit corridor at end of mission. Secondary goals are to perform several rendezvous, capture and docking scenarios as well as orbit maneuvers with the mated configuration.

\subsection{New Challenges}

Rendezvous and Docking is state of the art for manned spaceflight missions today. In addition to the new OOS applications, new technological requirements can be found for:

- Rendezvous phase

- Docking phase

- Degree of Autonomy

Typically, the target satellites have not been built for rendezvous and docking tasks. Therefore the rendezvous 
sensors and systems have to cope with completely uncooperative targets. The robotic based mechanisms have to ensure a safe and reliable gripping or docking at a target without any foreseen docking mechanisms. For missions without continuous contact to ground (typically LEO missions), the on-board autonomy plays an important role.

One of the challenges of such OOS missions is to ensure a safe and reliable Rendezvous and Docking (RvD) process. Especially this phase has to be analysed, simulated and verified in detail. Classical approaches e.g. numerical simulations deliver only limited results. Therefore new simulation procedures, tests and appropriate testing facilities have to be defined. They shall allow the simulating and testing of the entire RvD process (including the flight $\mathrm{HW}$ of GNC components and systems) under typical conditions of the space environment.

In fact, there have been several examples of such simulators for simulating rendezvous and docking operations of space systems. German Aerospace Center (DLR) developed a simulation facility called European Proximity Operations Simulator (EPOS), a former version of the new EPOS facility introduced in this paper, two decades ago for simulating satellite rendezvous operations (Krenn, 1999, see Fig. 1). The facility was used to support the testing of ATV and HTV rendezvous sensors. NASA/MSF developed an HIL simulator using a 6-DOF Stewart platform for simulating the Space Shuttle being berthed to the International Space Station (ISS) (Ananthakrishnan, 1996 and Roe, 2004). The Canadian Space Agency (CSA) developed for their SPDM (Special Purpose Dexterous Manipulator, a robotic arm system on ISS) a SPDM Task Verification Facility (STVF) using a giant 6DOF, customer-built, hydraulic robot to simulate SPDM performing contact tasks on ISS (Piedboeuf, 1999 and Ma, 2004). US Naval Research Lab used two 6-DOF robotic arms to simulate satellite rendezvous for HIL testing rendezvous sensors (Bell, 2003). China is also developing a dual-robot based facility to simulate satellite on orbit servicing operations (Xu, 2007). The unique features of the new EPOS facility, in comparison with those existing systems, are that it uses two heavy-payload industrial robots which can handle a payload up to $240 \mathrm{~kg}$ and it allows one robot to approach the other from 25-meter distance until zero distance.

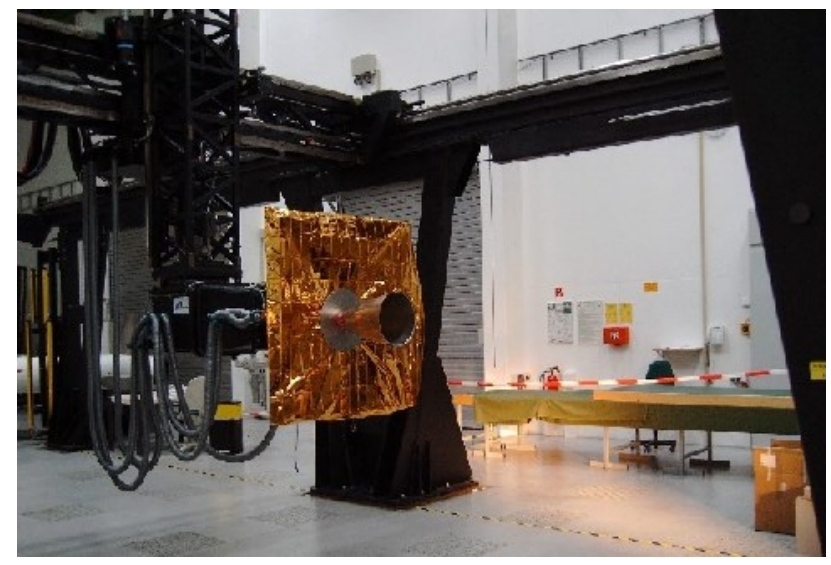

Fig. 1. The former EPOS facility.

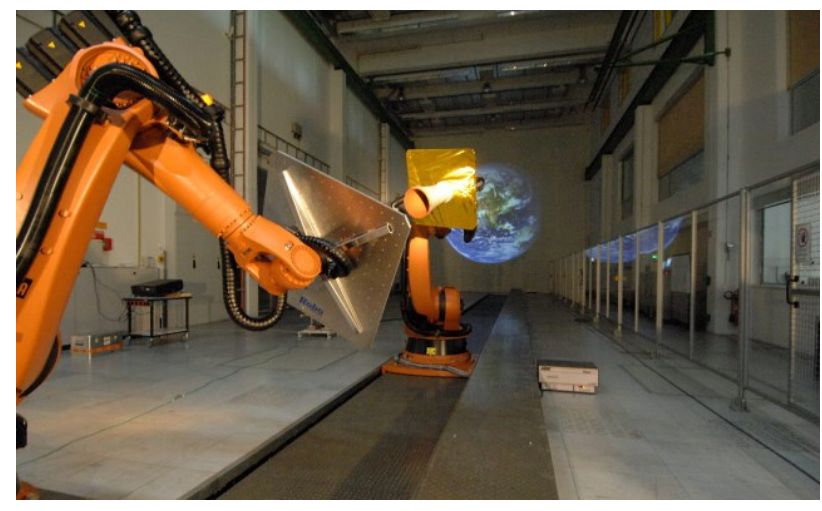

Fig. 2. The new EPOS 2.0 facility

\section{RENDEZVOUS AND DOCKING TEST FACILITY - EPOS 2.0}

\subsection{The New RvD Facilty - EPOS 2.0}

Future applications for satellite on-orbit servicing missions require the EPOS facility to be able to provide the following test and simulation capabilities

(A) the 6-DOF relative dynamic motion of two satellites in the final approaching phase from 25 to 0 meters.

(B) the 6-DOF contact dynamic behaviour during the entire docking process including the initial impact, soft docking, and hard docking (final rigidization).

(C) the space-representative lighting and background conditions

Since the old EPOS facility apparently could not provide all of these capabilities, it was completely replaced by a new EPOS 2.0 system. The design and construction work of the new facility began in 2008 .

The new EPOS 2.0 facility aims at providing test and verification capabilities for complete RvD processes of onorbit servicing missions. The facility comprises a hardwarein-the-loop simulator based on two industrial robots (of which one is mounted on a $25 \mathrm{~m}$ rail system) for physical realtime simulations of rendezvous and docking maneuvers. This test bed allows simulation of the last critical phase (separation ranging from $25 \mathrm{~m}$ to $0 \mathrm{~m}$ ) of the approach process including the contact dynamics simulation of the docking process.

Moreover, its main advances are:

- It is a highly accurate test bed. The measurement and positioning performance has been increased by factor 10 compared to the former EPOS facility.

- Dynamical capabilities allow for high commanding rates and the capability of force and torque measurements.

- The simulations of sunlight illumination conditions as well as the compensation of Earth-gravity force by advanced numerical simulation capabilities are both part of the assembly to generate an utmost realistic simulation of the real rendezvous and docking process. 


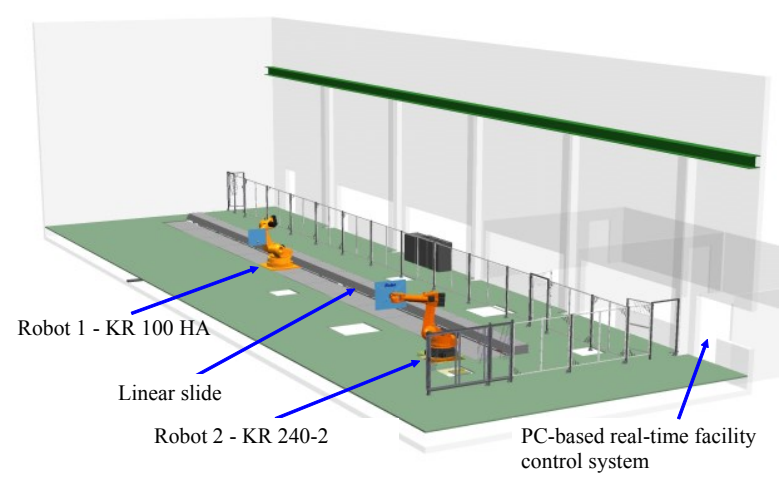

Fig. 3. Components of the new test bed - EPOS 2.0.

- The utilization of standard industrial robotics H/W allows a very high flexibility related to different application scenarios.

The new facility consists of two industrial robots: one is mounted on a $25 \mathrm{~m}$ rail system, the other one is fixed mounted at the end of the rail system which is shown in Fig. 3. Each of them can simulate the $6 \mathrm{DoF}$ of the servicer or client spacecraft. A system based on several PC's is used to monitor and control the entire facility.

\subsection{Capabilities and performances}

Table 1 summarizes the EPOS motion simulation capabilities and performances (Boge, 2009).

Table 1. EPOS motion capabilities

\begin{tabular}{lc}
\hline Parameter & Value \\
\hline Position: & $>20.0$ \\
Range [m] & $>1.0$ \\
Lateral [m] & \\
\hline Attitude: & $>600$ \\
Roll [deg] & $>150$ \\
Pitch, Yaw [deg] & \\
\hline Maximum velocity: & 2 \\
Translational [m/s] & 180 \\
Rotational [deg/s] & \\
\hline Command Interface: & 250 \\
Command rate [Hz] & $8-10$ \\
First natural frequency [Hz] & \\
\hline
\end{tabular}

Because EPOS is used for RvD sensor verification purposes, the facility was extensively calibrated after its installation. With a laser tracker device an overall positioning accuracy of the facility of better than $2 \mathrm{~mm}(3 \mathrm{D}, 3 \sigma)$ and an orientation accuracy of $0.2 \mathrm{deg}(3 \mathrm{D}, 3 \sigma)$ have been verified. In addition, it is planned to develop an online measurement system that measures the relative position between both robots and commands corrections to the robots. So the achieved position accuracy will eventually be in the sub-millimeter range. Furthermore, a lot of effort was made to increase the command frequency to $250 \mathrm{~Hz}$ which is an important precondition to simulate real-time contact dynamics.

\section{HARDWARE IN THE LOOP CONCEPT FOR RENDEZVOUS SIMULATION}

\subsection{Overview}

Hardware-in-the-loop simulation is a very effective way to perform verification and testing of complex real-time embedded systems like rendezvous sensors. Inputs and outputs of an embedded system are connected to a correspondent counterpart - the so-called HIL-simulator - that simulates the real environment of the system.

A typical HIL setting for rendezvous simulation is as follows: A rendezvous sensor for relative navigation measures relative position and attitude of the servicing satellite with respect to the target satellite. Based on this measurement thruster commands are computed by comparison of the actual position and attitude with the reference guidance values. Control commands for actuators like thrusters or reaction wheels cannot be simulated with real hardware. However the computation of forces and torques can be used to determine the position and attitude numerically based on equations of motion for the satellites' orbit and attitude. In the next sample, the computed positions and attitudes are commanded to the facility and are executed by the robots.

The main task in rendezvous simulation is to develop a stable control loop for orbit and attitude control. Figure 4. shows a typical control loop for a rendezvous scenario including sensor system, guidance, navigation and control functionality, actuators and the satellites' dynamics, kinematics and their environment.

The state, i.e. relative position and attitude, is simulated by the manipulators of the EPOS facility. The manipulator can be regarded as the connection of the numerical HIL-simulator with the embedded system, i.e. with the rendezvous sensor.

In the following section the dynamical models, the navigation sensor and the GNC system are described in detail. Finally, an overview on technical aspects concerning development of real-time rendezvous simulation software is given.

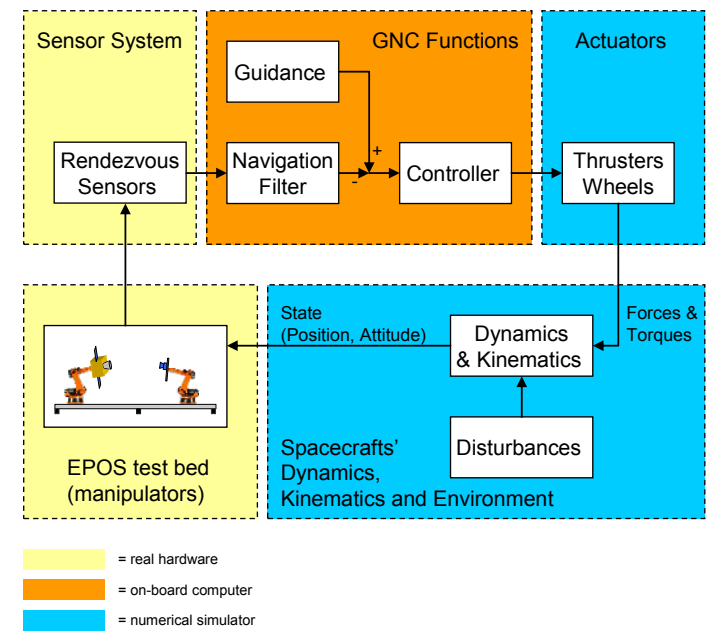

Fig. 4. Control loop for rendezvous. 


\subsection{Dynamical and kinematical spacecraft models}

The objective is to develop a realistic simulation of the rendezvous process including the real orbit mechanics. A numerical model is implemented to emulate the realistic motion of the satellites in orbit. For orbit control, position and velocity are calculated in the Clohessy Wiltshire (CLW) coordinate framework where the origin of the CLW coordinate framework is aligned with the center of mass of the target spacecraft.

The Hill equations (Fehse, 2003) are used to describe the chaser's relative translational motion in the local reference system of the target. The equations of motion are a system of linear ordinary differential equations:

$$
\begin{aligned}
& \ddot{x}=2 \omega_{0} \dot{z}+\frac{1}{m} f_{x}, \\
& \ddot{y}=-\omega_{0}^{2} y+\frac{1}{m} f_{y}, \\
& \ddot{z}=-2 \omega_{0} \dot{x}+3 \omega_{0}^{2} z+\frac{1}{m} f_{z},
\end{aligned}
$$

$f$ is the sum of control and disturbance forces acting on the chaser satellite. The activation of thrusters and the simulation of the real orbit mechanics are done numerically by solving the equations of motions. The computed position and attitude is then commanded to the facility.

The spacecraft attitude is described by Euler angles and quaternions. Here the following Euler angles convention is used. An orientation described by the angles $(\varphi, \theta, \psi)$ consists of three consecutive rotations: First a rotation around the $x$-axis with angle $\varphi$, then a rotation around the resulting $y$-axis with angle $\theta$ and finally a rotation around the resulting $z$-axis with angle $\psi$.

The attitude kinematics of chaser and target are each given by the quaternion differential equation (Wertz, 2002):

$$
\dot{q}=\frac{1}{2} \Omega(\omega) q,
$$

where

$$
\Omega(\omega)=\left(\begin{array}{cccc}
0 & \omega_{x} & -\omega_{y} & \omega_{z} \\
-\omega_{z} & 0 & \omega_{x} & \omega_{y} \\
\omega_{y} & -\omega_{x} & 0 & \omega_{z} \\
-\omega_{x} & -\omega_{y} & -\omega_{z} & 0
\end{array}\right)
$$

The attitude dynamics can be expressed by Euler equation (Wertz, 2002):

$$
I \dot{\omega}=T-\omega \times(I \omega) .
$$

Here, $T$ denotes the sum of control and disturbance torques. Both, quaternion and Euler equation are a system of nonlinear differential equations.

For solution of the orbit and attitude dynamic models Euler method with a time step of $4 \mathrm{~m}$ is used. This is the sample time the facility requires. The current configuration on EPOS restricts every Simulink application to use Euler method as solver for ordinary differential equations.

\subsection{Guidance Navigation and Control (GNC)}

The GNC functions are implemented as software in the automated onboard computer of the chaser satellite. They use the measurements delivered by a vision-based rendezvous sensor to calculate commands for the actuators (e.g. thrusters). A rendezvous approach requires continuous control of relative position and attitude.

The developed rendezvous simulation contains a guidance subsystem which provides reference values for the state at each sample time to generate a position and attitude profile. The objective of guidance is to define and force a state that the spacecraft should finally reach. Currently, several guidance modes are implemented which guide the servicer satellite toward the client.

For the navigation we use cameras which provide actual only $2 \mathrm{D}$ measurements. An image processing unit and/or software is necessary to provide full 3D pose estimation for the client with respect to the servicer satellite. Details to the navigation sensors are provided in chapter 4 where two different sensor and simulation concepts will be presented.

A navigation filter for orbit estimation is implemented and integrated in the control loop as shown in Fig. 4. The filter provides an estimate for the relative position and attitude of the servicer. The task of a navigation filter is to provide the controller with the necessary information about the current state of the spacecraft. The objective of all filters is to calculate an estimation of the state vector which is optimum by some measure. For rendezvous simulation described in this paper a Kalman filter is implemented which tries to minimize the variance of the estimation error.

To control the guided position trajectory a conventional PID controller is currently used. The corresponding controller gains are calculated based on the desired steady state performance requirements and the desired damping of the entire system. In future more advanced controllers will be designed and implemented to achieve the best system performance.

\section{SIMULATION RESULTS}

\subsection{Monocular CCD Camera Sensor}

As vision sensor a Prosilica Gigabit Ethernet vision camera (GC-655) has been used to measure the relative position and attitude (pose). In detail, it is a monochromatic, VGAresolution (640x480) charge coupled device (CCD) sensor 


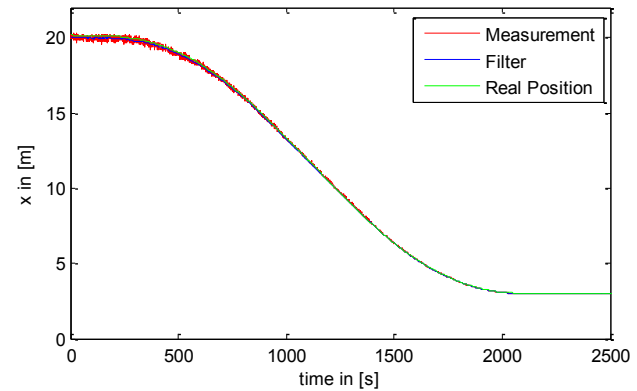

Fig. 5. x-coordinate (approach direction) of position measurement, filter estimates and real trajectory during an approach from $20 \mathrm{~m}$ to $3 \mathrm{~m}$

with large pixels on the chip, to increase sensitivity (Tzschichholz, 2010). The sensor chip has a very high dynamic range to cover various lighting situations.

Image processing algorithm then determine the pose of the target object in real-time. It is an algorithm to track a previously identified object. Since only a single camera is used, additional information about the target's geometry is necessary to obtain full 6-DoF pose estimation. I.e. in the tests performed at EPOS, a rectangular target with known edge lengths has been used.
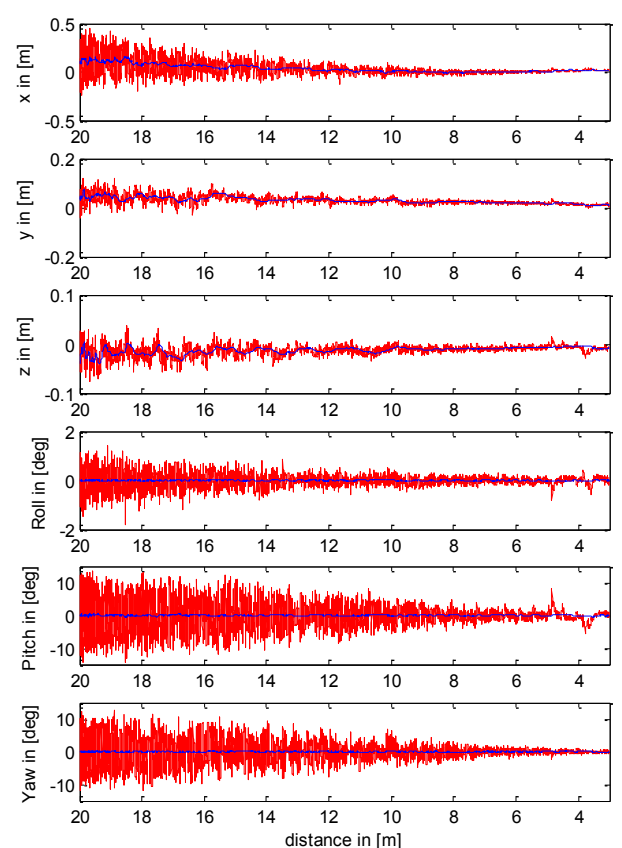

Fig. 6. Position and attitude error during an approach from $20 \mathrm{~m}$ to $3 \mathrm{~m}$

An approach from a distance of $20 \mathrm{~m}$ to a distance of $3 \mathrm{~m}$ between chaser and target has been simulated. The hold point of $3 \mathrm{~m}$ has been chosen due to the camera's field of view. The guidance function delivers a defined trajectory and forces a continuous approach. Fig. 5. shows the x-coordinate of the resulting position vector. Measurement and filter as well as the real position are plotted. In addition, Fig. 6. presents the error of measurement (red) and Kalman filter estimates (blue) with respect to the distance.
The closed-loop simulation delivers stable values during the entire approach. One can observe a significant decrease in the noise with decreasing distance to the target. However, even big noise is well smoothed by the filter at all distances.

\subsection{VIBANASS- VIsion BAsed Navigation Sensor System}

VIBANASS poses a versatile Rendezvous and Docking Camera System (CS), including a Target Illumination System (TIS), for LEO and GEO missions (Muehlbauer, 2012). It is set up with a camera subsystem with three radiation tolerant $1024 \times 1024$ pixel b/w sensors controlled by an electronics module which contains necessary control and power supply hardware and software including configurable image preprocessing. Three optical lenses will cover Close Range, medium range and Far Range distances, respectively. VIBANASS can be configured for mono and for stereo image acquisition, the latter one being used for short distances. The Far Range camera images are used for initial target identification starting from a few kilometres distance (depending on the true size) and for subsequent tracking until the Mid Range camera images become superior in image quality. Light conditions during the final approach and the docking phase are improved by activation and control of a laser-based Target Illumination System (TIS), thus improving the image quality for distance determination. An image processing algorithm has been developed for VIBANASS which provides 3D position measurements (Muehlbauer, 2012).

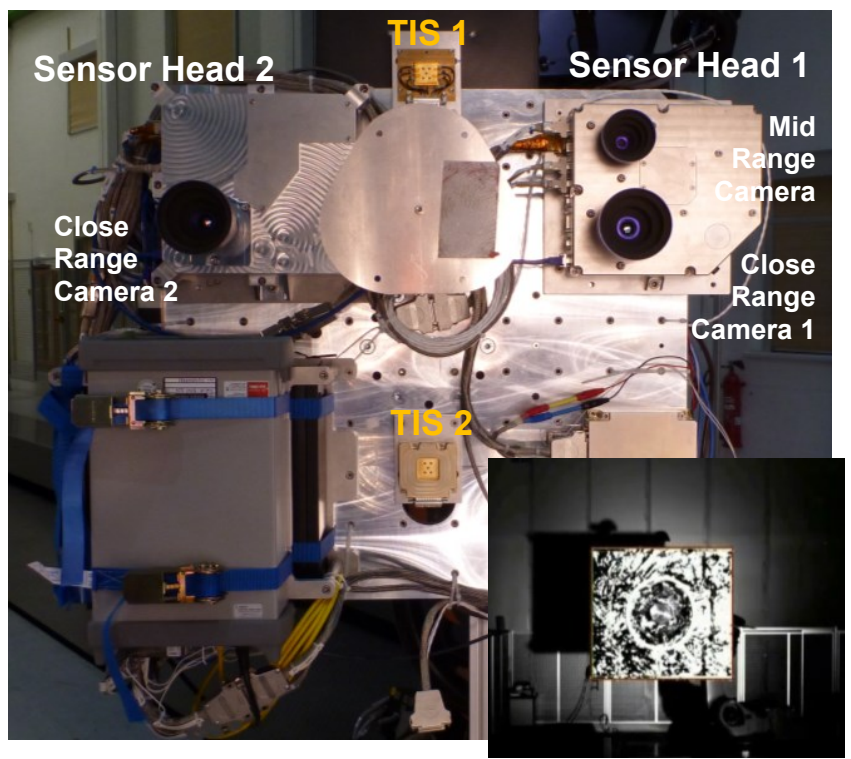

Fig. 7. Operational test configuration of VIBANASS and camera image of mid range camera

An approach from $18 \mathrm{~m}$ to $2 \mathrm{~m}$ was performed by using the image processing system as input for the navigation system which tracks the outer edges of the satellite at mid range and the nozzle ring of the apogee engine at close range. The guidance trajectory consists of a hold point at $18 \mathrm{~m}$, a continuous approach to $5 \mathrm{~m}$, a hold point at $5 \mathrm{~m}$, where a switch from mono (mid range) to stereo (close range) camera takes place, a second approach to a final hold point at $2 \mathrm{~m}$. 
Figure 8 shows for each component measurement, filter estimates and real position of the servicer for an approach in $\mathrm{x}$-direction (V-Bar), therefore $\mathrm{x}$ corresponds with the distance to the target.

At $\sim 80$ seconds the loop has been closed, i.e. the filter estimate has been fed to the controller. Due to measurement errors which do not have zero mean, the controller uses erroneous values for the actual position. This leads to performance errors. This is also the main source for the deviation between real position and filter values, since the filter can smooth the raw measurement data, but cannot correct biases. At $5 \mathrm{~m},(\sim 900 \mathrm{~s})$ the hold point is reached and a switch to stereo camera is accomplished. The measurement error is much smaller which results in a better performance which can clearly been seen considering e.g. the y- and zcoordinate in Figure 8. The systematic measurement errors are mainly caused by calibration inexactness and by limitation in the accuracy caused by sensor parameters, e.g. the resolution: At large distances changes in position of some centimeters cause only sub-pixel changes in the position of target in the image. Therefore, these changes are hard to recognize by image processing.
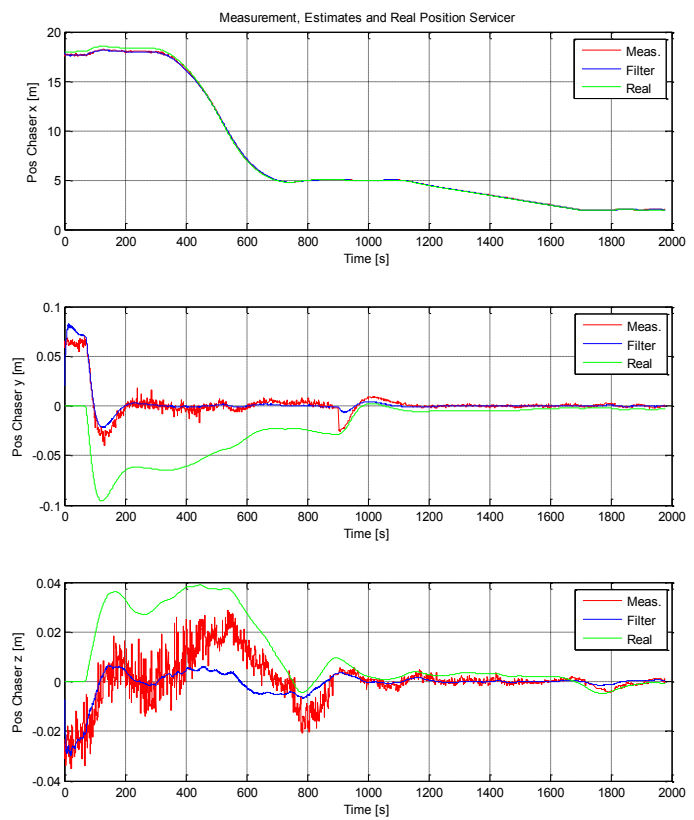

Fig. 8. Test result of VIBANASS closed loop test

\section{CONCLUSIONS}

This paper described the development of a rendezvous hardware-in-the-loop simulation including a vision-based sensor as well as the necessary robotics test bed EPOS 2.0. First tests of the closed-loop rendezvous simulation have been successfully executed in real-time for different projects. The dynamic behaviour during continuous approach from $20 \mathrm{~m}$ up to $2 \mathrm{~m}$ has been tested. The orbit and attitude control loop has been stable over the entire approach. The errors of the state estimation were below $1 \%$ with respect to the distance to the target which is acceptable for rendezvous navigation. So HIL simulation is a good technique to verify rendezvous processes for future OOS missions.

Future work focusses on other vision based sensors like LIDAR or PMD camera (Photonic Mixed Device). These sensors can provide more accurate distance estimation which could provide better navigation results in future.

\section{REFERENCES}

Ananthakrishnan, S., Teders R., and Alder, K., Role of estimation in real-time contact dynamics enhancement of space station engineering facility, IEEE Rob. and Auto. Mag., Vol. 3(3), 1996, pp. 20-28.

Bell, R., Morphopoulos, T., Pollack, J., Collins, J., Wertz, J. R., and Van Allen, R. E., Hardware-in-the-loop tests of an autonomous gn\&c system for on-orbit servicing, AIAA-LA Section/SSTC Responsive Space Conference 2003.

Boge, T., Rupp, Th., Landzettel, K., Wimmer, T., Mietner, Ch., Bosse, J., Thaler, B., Hardware-in-the-loop Simulator für Rendezvous- und Dockingmanöver. In Proceedings of DGLR-conference, 2009

Fehse, W., Automated rendezvous and docking of spacecraft, Cambridge Aerospace Series, Washington D.C., 2003.

Krenn, R. and Schaefer, B, Limitations of hardware-in-theloop simulations of space robotics dynamics using industrial robots, European Space Agency, ESA SP-440, Aug. 1999, pp. 681-686.

Ma, O., Wang, J., Misra, S., Liu, M., On the validation of SPDM task verification facility, J. of Rob. Syst., Vol.21(5), 2004, pp. 219-235.

Mühlbauer, Q., Richter, L., Kaiser, C., Hofmann, P., Robotics Space Systems and Subsystems for Advanced Future Programmes, I-SAIRAS, Turin, Italy, 4-6 September 2012

Mulder, T.A., Orbital Express autonomous rendezvous and capture flight operations, Part 1 of 2 and Part 2 of 2, AIAA/AAS Astrodynamics Specialist Conference and Exhibit, 18 - 21 August 2008, Honolulu, Hawaii

Piedboeuf, J.-C., De Carufel, J., Aghili, F., and Dupuis, E., Task verification facility for the Canadian special purpose dextrous manipulator, IEEE Int Conf on Rob and Auto, Detroit, 1999, Vol. 3, pp. 1077-1083.

Roe. F.D., Howard, R.T., and Murphy, L., Automated rendezvous and capture system development and simulation for NASA, Proc. SPIE, Vol. 5420, 118 (2004).

Rupp, Th., Boge, T., Kiehling, R., and Sellmaier, F., Flight dynamics challenges of the German on-orbit servicing mission DEOS. Proc. 21th ISSFD conference, 2009.

Tzschichholz, T. and Boge, T., GNC systems development in conjunction with a RvD hardware-in-the-loop simulator, 4th ICATT Conference, Madrid, Spain, 2010.

Wertz, J. R., Attitude determination and control, Kluwer Academic Publishers, Dordrecht, Boston, London, 2002.

Xu, W. F., Liang, B., Xu, Y. S., Li, C., and Qiang, W. Y., A ground experiment system of free-floating space robot for capturing space target, J. of Intell. and Rob. Syst., Vol.48(2), pp. 187-208, 2007. 